\title{
A Factor in Heat-Treated Mulberry Leaves That Inhibits Metamorphosis in Bombyx mori: Effects on Endocrine Regulation during Larval Development
}

\author{
Seiji TanaKa, ${ }^{1}$ Makoto Kiuchi and Mitsumasa Sudo \\ Department of Insect Physiology and Behavior, National Institute of Sericultural and \\ Entomological Science, Ohwashi 1-2, Tsukuba, Ibaraki 305, Japan
}

(Received May 25, 1989)

\begin{abstract}
High-temperature $\left(615^{\circ} \mathrm{C}\right)$ treated leaves of the mulberry tree, Morus alba L., contain a factor (mulberry factor, MF) that causes 5 th instars of the silkmoth, Bombyx mori $\mathrm{L}$. (Lepidoptera: Bombycidae), to become permanent larvae (Sudo and TANAKA, 1989). When allatectomized 5th instars were reared on a diet containing those leaves (HT diet), they became permanent larvae, as did sham-operated and intact controls. This suggests that the action of MF is not mediated through modifying the activity of corpora allata. Ecdysteroid titers in hemolymph, which began to increase within a week after molting to the 5 th instar on a standard diet, remained at a low level longer than 2 weeks both in allatectomized and intact or sham-operated larvae kept on the HT diet. This reduced ecdysteroid titer may be the main cause for induction of permanent larvae. In organ culture, prothoracic glands isolated from 5-day old 5th instars reared on the HT diet were found competent to respond to prothoracicotropic hormone (PTTH) by secreting ecdysone into the medium. It is thus likely that MF caused low ecdysteroid titers in hemolymph by suppressing secretion of PTTH from the brain. When 4 th instars were reared on the HT diet, growth and the hemolymph ecdysteroid titer were not altered prior to the molt to the 5 th instar.
\end{abstract}

\section{INTRODUCTION}

Leaves of the mulberry tree, Morus alba, that have been dried at a very high temperature, i.e. $615^{\circ} \mathrm{C}$, contain some factor or substance(s) that causes larvae of the commercial silkmoth, Bombyx mori, to become permanent larvae (Sudo and AkAI, 1988; Sudo and TANAKA, 1989). This factor prevents 5 th instar larvae from spinning cocoons and stimulates their continuous feeding, resulting in production of huge permanent larvae. Larvae kept on a diet containing this mulberry factor (MF) during the first few days of the 5 th instar spin cocoons and pupate when transferred to a standard diet which contains mulberry leaves treated in a conventional manner, i.e. $100^{\circ} \mathrm{C}$ (Supo and TANAKA, 1989). In this case, cocoons and pupae are significantly larger than those produced on the standard diet alone. Apparently, the effects of MF on growth and metamorphosis are reversible as long as a therapy treatment, i.e. a transfer to a normal diet, is done before a critical period.

It is interesting that permanent larvae can also be produced by application of juvenile hormone or its analogues in B. mori (Akai and Kobayashi, 1971; Akai et al., 1973; Kajiura and Yamashita, 1989; Kajiura et al., 1987; Sakurai and Imokawa,

1 To whom all correspondence should be addressed. 
1988). As demonstrated for JH-treated individuals (Kajiura et al., 1987; Akai et al., 1988), permanent larvae produced with MF can be stimulated to spin a cocoon by application of 20-hydroxyecdysone (Sudo and AKaI, 1988). It is thus likely that MF induces permanent larvae through acting on the endocrine system in $B$. mori. The present study explores this possibility. The first hypothesis we test is that MF influences metamorphosis of $B$. mori by elevating the activity of the corpora allata, the endocrine glands that produce juvenile hormone. We then test if MF suppresses the ecdysteroid titer in hemolymph so that larval-pupal metamorphosis is inhibited. We also examine how such suppression, if it occurs, is brought about: Does MF act on the prothoracic glands or the brain that produces prothoracicotropic hormone (PTTH)? The present paper will describe and discuss the results of our study testing these hypotheses.

\section{MATERIALS AND METHODS}

Larvae of $B$. mori $\left(\mathrm{J} 02 \times \mathrm{C} \mathrm{02}\right.$ ) were reared on artificial diets at $25 \pm 1^{\circ} \mathrm{C}$ and 12L-12D. Two types of diets were prepared according to the recipe of Sudo and AkaI (1988). Both contained the same amount of dried leaves of $M$. alba (MinamiSAKARI) and differred from each other only in the way the leaves were dried. One contained leaves dried at a relatively low temperature $\left(100^{\circ} \mathrm{C}\right)$ while the other contained those dried briefly at a high temperature $\left(615^{\circ} \mathrm{C}\right.$ ) (Sudo and AkaI, 1988). As defined in a previous work (SUDo and TANAKA, 1989), they will be called the LT diet and HT diet, respectively. The LT or standard diet (containing low-temperature treated leaves) does not cause abnormal development in $B$. mori while the HT diet (containing high-temperature treated leaves) has been demonstrated to induce permanent larvae at the 5 th instar when fed either from the beginning of the first instar or from that of the 5th instar. In the present study, only males were used because of possible differences in result between the sexes.

To test the hypothesis that MF elicits its effect on the development of $B$. mori through action on the corpora allata, we removed the corpora allata from larvae during the fourth instar. Shortly before their head capsule became pulled away from the pronotumn (head capsule slippage), larvae were lightly anesthetized with ethyl ether and the corpora allata removed through small incisions made on the ventral side of the neck. Most allatectomized larvae showed head capsule slippage by the following day and molted to the 5 th instar within 2 days after surgery. Such larvae can complete development if kept on the LT diet and, upon adult emergence, they mate and lay fertile eggs (KIUchI, unpublished observations). Upon reaching the 5th instar, allatectomized larvae were fed either the LT diet or the HT diet. As controls, intact and shamoperated larvae were also reared under the same conditions. Sham-operated larvae received all the surgical procedure for allatectomy except that their corpora allata were left intact. All larvae were given fresh diet every day and checked for spinning behavior and pupation as described in a previous paper (Sudo and TANAKA, 1989). Individual body weight was daily recorded.

To determine how MF influences the ecdysteroid titer in hemolymph of 5th instars with or without corpora allata, allatectomized, sham-operated, and unoperated larvae were reared on the HT diet in the same way as in the above experiment. For comparison, a group of unoperated 5th instars were reared on the LT diet. From each group 3 larvae were removed on each of different occasions during the 5 th instar and 
$100 \mu \mathrm{l}$ of hemolymph was withdrawn from each individual. The hemolymph samples thus collected were stored at $-20^{\circ} \mathrm{C}$ until used. Titers of ecdysteroids in hemolymph samples were determined by a radioimmunoassay using 20-hydroxyecdysone (Sigma) as a standard according to the method described by TAKEDA et al. (1986).

To determine if $\mathrm{MF}$ affects the competence of prothoracic glands to respond to PTTH, prothoracic glands removed from 5-day old 5th instars were incubated in GrAGE's insect culture medium (GIBCO Laboratories, Grand Island, NY) with or without addition of a partially purified sample of $22 \mathrm{~K}$-prothoracicotropic hormone (a gift from Dr. H. NAGAsawa of Tokyo University) according to the method of Okuda et al. (1985). After a 6-hour incubation at $20^{\circ} \mathrm{C}$, the amount of ecdysone released into the medium was determined by a radioimmunoassay.

To test if MF influences the ecdysteroid titer of hemolymph before the 5th instar, male larvae reared on the LT diet until the end of the 3rd instar were kept either on the HT diet or the LT diet during the 4 th instar at $25^{\circ} \mathrm{C}$ and $12 \mathrm{~L}-12 \mathrm{D}$. Changes in body weight and duration of the 4th instar were determined for 50 individuals on each diet. Hemolymph samples were collected every 12 hours and their ecdysteroid titers determined as described above.

\section{RESULTS}

Intact or sham-operated larvae reared on the LT diet began to spin silk within a week after molting to the 5th instar (Fig. 1A). They took an additional 3-5 day period to pupate and showed no statistical difference in duration of the 5th instar. Allatectomized larvae also developed in a similar way but they tended to produce silk earlier and died earlier than did the other groups, although the differences were not statistically significant ( $t$-test, $p>0.05$ ). When intact and sham-operated larvae were reared on the HT diet (Fig. 1B), some started spinning 3 weeks after molting to the 5 th instar, but the rest died without spinning silk at all. Allatectomy did not influence development of the 5th instar and no statistical difference was detected in duration of the 5th instar between groups on the HT diet $(p>0.05)$. However, the proportion of individuals spinning silk $(72 \%)$ in the allatectomized group was significantly greater than that in the intact group $\left(24 \%, \chi^{2}=9.67 ; p<0.05\right)$ or sham-operated group $(31.8 \%$, $\left.\chi^{2}=6.12 ; p<0.05\right)$, although no larvae constructed cocoons and pupated on this diet. These results indicate that corpora allata are not responsible for induction of permanent larvae by MF.

Figure 2 illustrates changes in body weight of 5 th instars reared on the LT diet (A) or the HT diet (B). As already demonstrated for females by Sudo and TANAkA (1989), male larvae kept feeding longer and grew larger on the HT diet than on the LT diet. Allatectomized larvae tended to be smaller than intact or sham-operated ones. However, the pattern of changes in body weight was the same for all groups on each diet.

The ecdysteroid titer of intact 5 th instars on the LT diet reached about $50 \mathrm{ng} / \mathrm{ml}$ hemolymph on day 6 when they were about to spin silk (Fig. 3). The titer further increased and reached a high level one day before pupation. Intact and allatectomized larvae on the HT diet, on the other hand, showed very low ecdysteroid titers for more than 2 weeks after molting to the 5 th instar (Fig. 3). When spining behavior began, the titers increased to about $50 \mathrm{ng} / \mathrm{ml}$ hemolymph. The results for sham-operated larvae 
(A) 10

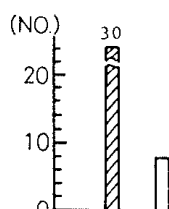

Duration of

Intact

$10.7 \pm 0.4(30)($ days $)$

(B)

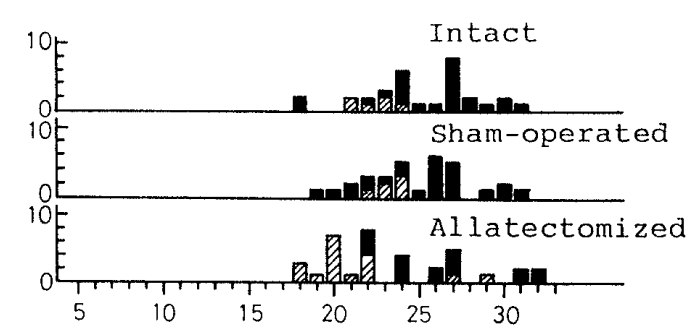

$25.6 \pm 3.2(25)$

$25.0 \pm 2.8(22)$

$25.0 \pm 3.5(27)$

DAYS AFTER MOLTING TO THE 5TH INSTAR

Fig. 1. Development of 5th instar larvae in B. mori on the LT diet (A) or HT diet (B) at $25^{\circ} \mathrm{C}$. All larvae were reared on the LT diet before reaching the 5 th instar. Two days before molting to the 5 th instar, larvae were either allatectomized, sham-operated, or left unoperated. Hatched, open and closed columns indicate the numbers of animals that initiated spinning, pupated, and died as larvae, respectively. Numbers in parentheses indicate the numbers of individuals observed. Duration of the 5th instar is reported as the period of time during which animals lived as 5th instars.
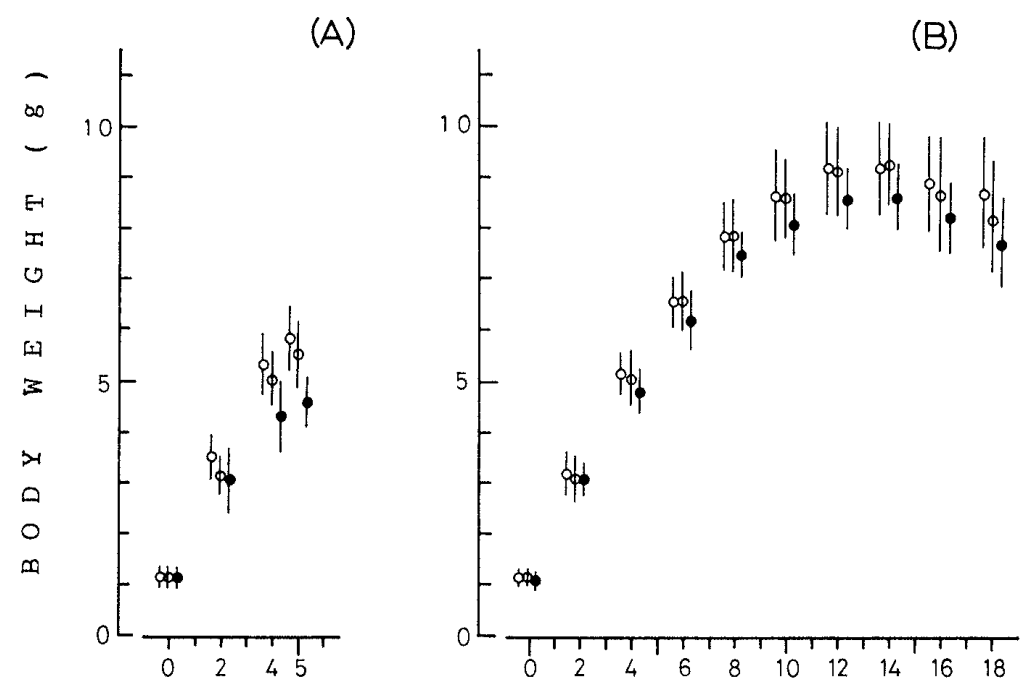

DAYS AFTER MOLTING TO THE 5TH INSTAR

Fig. 2. Changes in body weight (Mean \pm standard deviation) of 5 th instar larvae in B. mori on the LT diet (A) or HT diet (B) at $25^{\circ} \mathrm{C}$. Two days before molting to the 5 th instar, larvae were either allatectomized $(\bullet)$, sham-operated $(\mathbb{D})$ or left unoperated $(0)$. Data were collected from the same individuals as those in Fig. 1. 


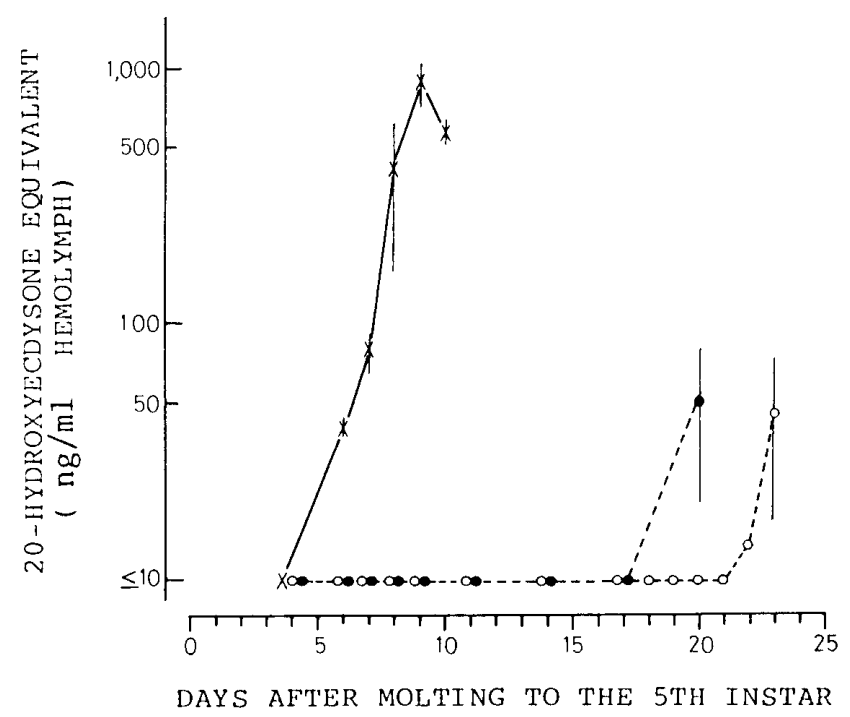

Fig. 3. Changes in hemolymph ecdysteroid titer (Mcan \pm standard deviation) of 5th instar larvae in B. mori on the LT diet (crosses) and the HT dict (circles) at $25^{\circ} \mathrm{C}$. Closed circles indicate the results for larvae allatectomized two days before molting to the .5th instar. For developmental events observed under the same conditions, see Figs. 1 and 2.

Table 1. Effects of prothoracicotropic hormone (PTTH) on ecdysteroid production by prothoracic glands isolated from 5-day old 5 th instars in $B$. mor $i^{\text {a }}$

$\begin{array}{lccc}\text { Treatment } & \begin{array}{c}\text { Control glands } \\ \text { (Without PTTH) } \\ \text { (ng 20-hydroxyecdysone equivalent/gland/6 hr) }\end{array} & \begin{array}{c}\text { Experimental glands } \\ \text { (With PT'H) }\end{array} & \text { l-test } \\ \text { LT diet }(N=5) & 3.60+1.06^{1} & 10.57 \pm 2.47^{\mathrm{h}} & * * \text { * } \\ \text { HT diet }(N=6) & 0.67 \pm 0.17 & 7.46 \pm 1.57 & * *\end{array}$

a Larvae were kept either on the HT diet or LT diet after molting to the 5 th instar at $26^{\circ} \mathrm{C}$. One prothoracic gland of a pair was incubated in $200 / 1$ of Grace's medium alone (control gland) and the contralateral one with one head equivalent of PTT'H (expcrimental gland).

1. Mean \pm standard deviation.

"Significant at $5 \%$ both between control and experimental glands and between treatments.

on the HT diet are not shown here because they were almost identical to those obtained from intact larvae on the same diet. These results clearly indicate that the H'T diet strongly suppresses the hemolymph ecdysteroid titer during the 5 th instar and this could be the main reason for induction of permanent larvae.

An organ culturing method was adopted to determine if prothoracic glands from larvae reared on the HT diet were competent to produce ecdysone in response to a hormonal stimulus (PTTH) (Table 1). As inferred from Fig. 3, the glands were inactive in terms of ecdysone production as compared with the glands from larvae kept on the LT diet. However, the inactive glands showed about a 10 -fold increase in ecdysteroid production when incubated with PTTH. Although the absolute level of ecdysone production was still lower than that for larvae kept on the L'T' diet, the results 


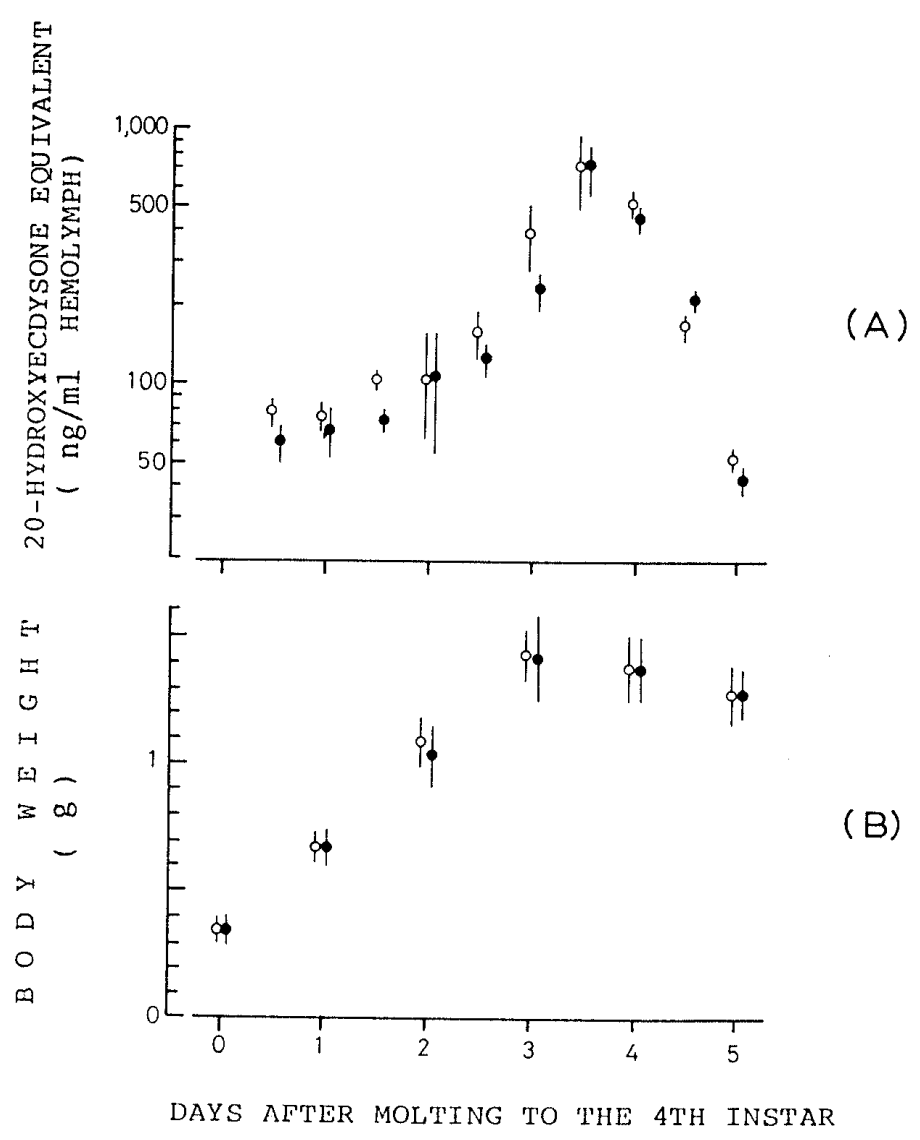

Fig. 4. Changes in hemolymph ecdysteroid titer (A) and body weight (B) of 4th instar larvae in B. mori on the LT diet (open circles) and the HT diet (closed circles). Each point indicates a mean of 3 and 50 larvae in (A) and (B), respectively. Vertical lines indicate standard deviation of the means.

clearly indicate that the prothoracic glands of larvae reared on the HT diet are capable of producing ecdysone in the presence of PTTH.

Figure 4 shows patterns of growth and changes in ecdysteroid titer during the 4th instar during which larvae were kept on the HT diet or the LT diet. Larvae kept on the HT diet $(5.5 \pm 0.5$ days; $n=50)$ took slightly longer to complete development of the 4 th instar than those reared on the LT diet $(5.0 \pm 0.0$ days; $n=50)$. Yet, the two groups showed almost identical patterns both in growth and ecdysteroid titer.

\section{DISCUSSION}

Induction of permanent larvae has been reported for $B$. mori by several groups of workers. Akai and Kobayashi (1971) obtained permanent larvae by injecting 5th instars with small dosages of JHA, i.e. $1 \mu \mathrm{g} /$ animal. Sakura and Imokawa (1988) have confirmed this and suggested that JHA affects both the brain and prothoracic glands in such a way that the hemolymph ecdysteroid titer remains at a low level. Permanent larvae obtained in this way show reduced feeding activity and their body size remains small until they die. It is known, however, that if larvae receive JHA 
topically either during the 4th instar (KAJIURA et al., 1987) or during the 5th instar (Akar et al., 1988), they become large permanent larvae after a long period of feeding during the 5th instar. Another difference observed is that small permanent larvae induced by injection of JHA undergo larval-larval molting in response to administration of 20-hydroxyecdysone (SAkuraI and Imokawa, 1988) while large permanent larvae produced by topical application of JHA respond by spinning cocoons. Interestingly, the former do pupate if injected with PTTH instead of 20-hydroxyecdysone (SAKURAI and IMOKAwA, 1988). Although persistently reduced ecdysteroid titers in the hemolymph may explain induction of permanent larvae in both cases (KAJIURA et al., 1987; SAKURAi and Imokawa, 1988), the above differences suggest the complicated nature of endocrine regulation in this insect.

Permanent larvae produced on the HT diet containing MF appear to be similar to those induced by topical application of JHA. The permanent larvae induced by MF show an extended period of feeding and attain a maximum body weight $50-100 \%$ heavier than normal individuals (Sudo and AkAI, 1988; Sudo and TANAkA, 1989; Fig. 2 in this study). According to Kajiura et al. (1987), JHA suppresses the ecdysteroid titer, thus preventing larval-pupal metamorphosis. Furthermore, permanent larvae can be induced by JHA even among allatectomized individuals. Therefore, JHA action is not mediated through modifying the activity of corpora allata. However, how such a reduced ecdysteroid titer is brought about and maintained by JHA remains unknown.

In the present study, permanent larvae were induced in both intact and allatectomized larvae on the HT diet. Therefore, MF in the HT diet induces permanent larvae by affecting something other than the activity of corpora allata, although allatectomy influences the timing of the initiation of spinning behavior as well as the proportion of individuals spinning silk. As observed for JHA-treated individuals (KAJIURA et al., 1987), larvae on the HT diet show a persistently reduced ecdysteroid titer while a rapid increase in the ecdysteroid titer is observed in larvae on the LT diet. This, together with the fact that these permanent larvae can be stimulated to spin cocoons by administration of 20-hydroxyecdysone (SUdo and AKaI, 1988), suggests that deficiency of ecdysteroids in the hemolymph is also the cause for failure to undergo larvalpupal metamorphosis of larvae reared on the HT diet.

How MF causes low ecdysteroid titers in hemolymph is an interesting question. The present results show that the prothoracic glands isolated from larvae reared on the HT diet remain competent to produce ecdysone in response to PTTH. Therefore, it is possible that MF in the HT diet induces low ecdysteroid titers in hemolymph by inhibiting production and/or secretion of PTTH from the brain. In vivo experiments to test this possibility have not yet been carried out. An additional mechanism possibly contributing to the persistance of low ecdysteroid titers is an inhibitory action of MF on the activity of the prothoracic glands. This hypothesis is based on the finding by SAKURAI (1984) that when the brain together with the corpora cardiaca and corpora allata was removed from 4-day old 5th instars, the prothoracic glands in those larvae became spontaneously active several days later in B. mori. From this fact, Sakurai and ImokAwA (1988) have also concluded that JHA affects not only the brain but also the prothoracic glands. In their study, however, how long JHA persisted in larvae and how it affected the activity of corpora allata have not been determined.

Although MF and JHA appear to show some similarities in action, they differ 
from each other in that MF needs to be fed continuously to prevent larvae from spinning cocoons and pupating while a single application of JHA can induce permanent larvae. Thus, larvae on the HT diet can be prevented from becoming permanent larvae by transferring them to the LT diet (Sudo and TANAKA, 1989). JHA shows various effects on growth and metamorphosis even when applied during early larval development (e.g. KajIura et al., 1987), but MF does not and its effect is not manifested before the last (5th) instar (Fig. 3). Therefore, it seems likely that MF is not another JHA, but chemical characterization is essential to clarify this point.

\section{ACKNOWLEDGEMENTS}

We are grateful to Yoshiteru Higashi for preparing first instars of B. mori, Noriko Kenmochi for her excellent technical assistance, and David L. Denlinger for reviewing the manuscript. Many thanks are also due to Satoshi TAKeda for valuable suggestions during the early stage of this work, and to Hiromichi NAGASAWA for a partially purified sample of 22K-prothoracicotropic hormone.

\section{REFERENCES}

Akai, H. and M. Kовayashi (1971) Induction of prolonged larval instar by the juvenile hormone in Bombyx mori L. (Lepidoptera: Bombycidae). Appl. Ent. Zool. 6: 138-139.

Akat, H., K. Kiguahi and K. Mori (1973) The influence of juvenile hormone on the growth and metamorphosis of Bombyx larvae. Bull. Seric. Exp. Stn. 25: 287-305.

Akai, H., K. TAKABayashr and M. Кivahi (1988) Induction of spinning from JH- treated eternal larvae of Bombyx mori by ecdysteroid administration. J. Seric. Sci. Jpn. 57: 341-344.

KajIura, Z. and O. Yamashita (1989) Super growth of silkglands in the dauer larvae of the silkmoth, Bombyx mori, induced by a juvenile hormone analogue. J. Seric. Sci. Jpn. 58: 39-46.

Kajiura, Z., K. Kadono-Okuda and O. Yamashita (1987) Induction of dauer larvae by a juvenile hormone analogue and their response to ecdysteroids in the silkmoth, Bombyx mori. J. Seric. Sci. Jpn. 56: 398-406.

Okuda, M., S. SAkurai and T. Ohtakr (1985) Activity of the prothoracic gland and its sensitivity to prothoracicotropic hormone in the penultimate and last-larval instar of Bombyx mori. J. Insect Physiol. 31: $455-461$.

SaKURAI, S. (1984) Temporal organization of endocrine events underlying larval-pupal metamorphosis in the silkworm, Bombyx mori. J. Insect Physiol. 30: 657-664.

Sakurai, S. and H. Imokawa (1988) Developmental arrest induced by juvenile hormone in larvae of Bombyx mori. Arch. Insect Biochem. Physiol. 8: 219-228.

Sudo, M. and H. AKaI (1988) Induction of spinning from non-spinning larvae reared with an artificial diet by administration of molting hormone. J. Seric. Sci. Jpn. 57: 405-408 (in Japanese).

Sudo, M. and S. TANAKA (1989) A factor in heat-treated mulberry leaves that inhibits metamorphosis in Bombyx mori: the effects on development of last instar larvae and pupae. Appl. Ent. Zool. 24: $441-449$.

TAKedA, S., M. KIUCHI and S. UEdA (1986) Preparation of anti-20-hydroxyecdysone serum and its application for radioimmunoassay of ecdysteroids in silkworm hemolymph. Bull. Seric. Exp. Stn. 30: 361-373 (in Japanese). 